\title{
Una obra del pintor flamenco Sebastiaen van Aken en España: el altar de Santa Cecilia en el convento de las Trinitarias Descalzas de Madrid*
}

\author{
A Painting by the Flemish Artist Sebastiaen van Aken in Spain: Saint \\ Cecilia's altar piece at the Convent of the Trinitarias Descalzas of Madrid
}

\section{Gloria Martínez Leiva ${ }^{1}$}

Resumen: A finales del siglo XVII llegaba a España el pintor flamenco Sebastiaen van Aken (1648-1722). De su actividad en nuestro país no se tenía ninguna noticia hasta el momento. Gracias a que dejó su firma en una de las obras que realizó, la cual hasta ahora no había sido reconocida, se le ha podido dar la autoría del cuadroaltar de Santa Cecilia que preside una de las capillas de la Iglesia del Convento de las Trinitarias Descalzas de Madrid.

Palabras clave Santa Cecilia, Sebastiaen van Aken, Iglesia del Convento de las Trinitarias Descalzas, pintura flamenca, capillas.

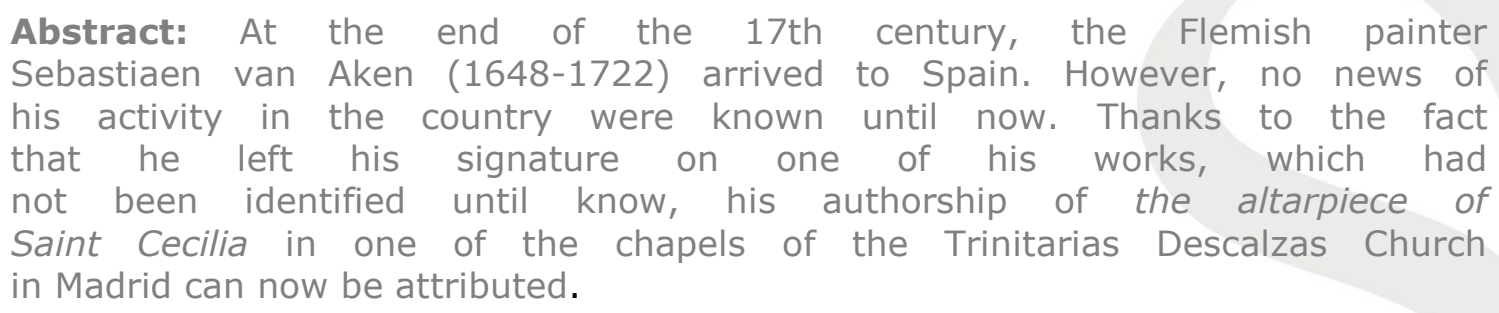

Key-words: Saint Cecilia, Sebastiaen van Aken, Convent's church of Trinitarias Descalzas, Flemish painting, chapels.

\footnotetext{
* Quiero agradecer a mi amiga y compañera la Dra. Ana Diéguez su apoyo e indicaciones a la hora de realizar este texto.

${ }^{1}$ http://orcid.org/0000-0002-7388-3989

(C) 2018 Philostrato. Revista de Historia y Arte
} 


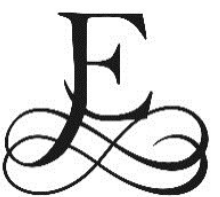

I 4 de septiembre de 1697 tenía lugar una misa solemne, oficiada por el Cardenal Portocarrero ${ }^{2}$, con la que se inauguraba la iglesia del Convento de las Trinitarias Descalzas de Madrid. El convento había sido fundado a principios del siglo XVII, el 17 de octubre de 1612, por Doña Francisca Gaitán Romero, mujer de gran fortuna. Tras la ruptura entre la fundadora y las religiosas debido a sus diferentes caracteres, éstas quedaron sin fondos suficientes para terminar la obra. Es por ello que tan sólo pudo construirse una modesta iglesia adyacente en la que se enterraron, por ejemplo, los restos de Miguel de Cervantes el 23 de abril de 1616.

No obstante, la buena ubicación del convento hará que muchas personalidades importantes se interesen y vinculen a éste, hasta que en 1630 se establezca un nuevo patronato para la iglesia y convento en la persona de María de Villena y $\mathrm{Melo}^{3}$, dama de la casa de Braganza, casada con un hijo del Duque de Medinaceli, por lo que desde ese momento el patronato de la iglesia y convento lo ejercerán los Duques de Medinaceli y los Marqueses portugueses de Aronches. Ya hacia mediados del siglo XVII se comenzó a pensar en levantar una nueva iglesia más acorde con el convento, pero hasta 1673 el Duque de Medinaceli no ajusta "la obra y su iglesia con Marcos López y su hijo José López"4. Marcos López había comenzado su trayectoria profesional como simple cantero, pero con el tiempo llegará a convertirse en uno de los tracistas más destacados del Madrid de la segunda mitad del siglo XVII ocupándose de obras como la Cárcel de Corte o participando en la capilla de San Isidro ${ }^{5}$. La antigua iglesia será derribada, al igual que algunas manzanas de casas cercanas, para así el 16 de septiembre de 1673, colocar la primera piedra del nuevo edificio ${ }^{6}$. Las obras fueron avanzando hasta que en 1688 , tras la muerte del arquitecto, éstas quedaron paralizadas y no será hasta el 26 de mayo de 1693, cuando se firme un nuevo concierto con el arquitecto José de Arroyo para que éste termine el proyecto que estaba hecho "hasta el sobrelecho de la cornisa en redondo"7. No obstante, sabemos que desde al menos 1690 ya se estaba planeando la construcción interior de algunas de las capillas del templo. De ello tenemos constancia gracias a un documento presente en el archivo del convento de Trinitarias fechado el 13

\footnotetext{
2 Virginia Tovar Martín, "El Monasterio de las Religiosas Trinitarias Descalzas de San Ildefonso de Madrid", Archivo Español de Arte, 251, (1990), p. 405.

3 Ramón Menéndez Pidal, "El convento de las Trinitarias de Madrid", Boletín de la Real Academia de la Historia, 79, (1921), p. 98.

${ }^{4}$ Virginia Tovar Martín, Arquitectos madrileños de la Segunda Mitad del siglo XVII, (Madrid: Instituto de Estudios Madrileños, 1975), p. 328.

${ }^{5}$ Sobre Marcos López y las obras que ejecutó véase Virginia Tovar Martín, "El arquitecto Marco López y el Convento de las Trinitarias Descalzas de Madrid", Anales del Instituto de Estudios Madrileños, 10, (1974), pp. 133-153 y Tovar Martín, Arquitectos madrileños, pp. 321-355.

6 Tovar Martín, Arquitectos madrileños, p. 328.

7 Tovar Martín, Arquitectos madrileños, p. 329.
} 


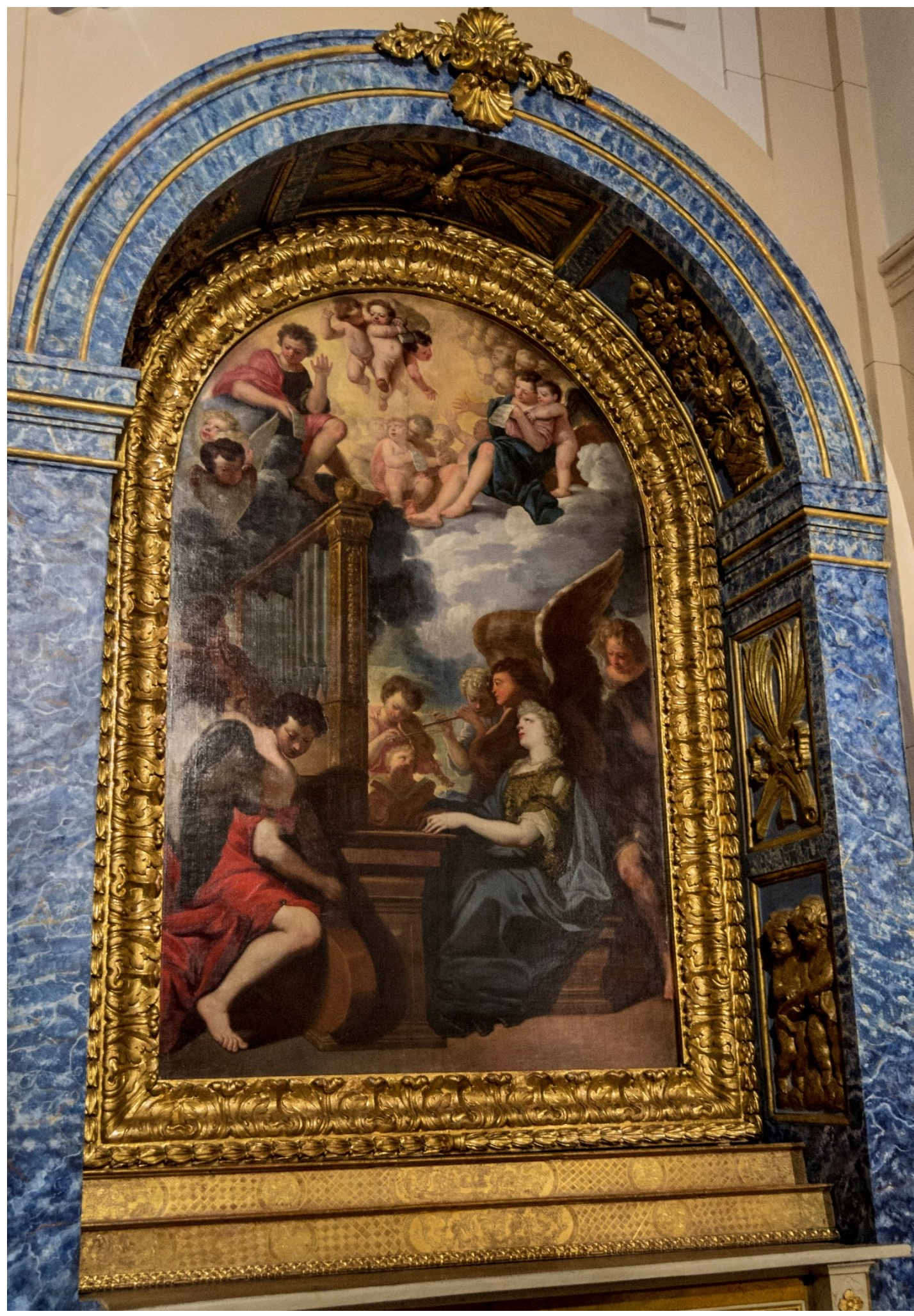

Fig. 1. Sebastiaen van Aken, Santa Cecilia, ca. 1697-1701. (o/1., 309 x 203 cm.) Madrid, Iglesia del Convento de las Trinitarias Descalzas de Madrid. Fotografía: Jesús Calleja Vicente $(\underset{C}{ }$ 
de marzo de 1690 y donde se dice "En el año de 1690 nos dio de limosna Nuestro Reverendísimo Padre General Fray P[edro] de San Miguel una capilla entera de Sta. Cecilia en el mes de marzo a 13 y siendo ministra Nuestra Madre Sor Antonio [sic] de Jesús"8.

Las capillas que se ejecutaron en la iglesia fueron cuatro, situadas a lo largo de la nave del templo, de planta de cruz latina, y en forma de capillashornacina presididas por un cuadro de altar. Éstas están dedicadas a San Felipe Neri, al Santo Cristo de Burgos, a San Agustín y a Santa Cecilia. La dedicada a Santa Cecilia, patrona de la música, se encuentra en el segundo tramo de la nave, en el lado de la Epístola, y está decorada con un cuadroretablo inscrito dentro del arco de medio punto, a modo de arcosolio, que configura la capilla. Sabemos gracias al Libro sobre la Fundación e Historia del Convento que el cuadro que la adorna "fue a devoción del Señor Dn. Diego de Mendoza y Corterreal, enviado extraordinario de Portugal"9 y "se celebró su colocación con misa cantada y asistencia de la Capilla de las Descalzas Reales"10. Éste no fue el único altar que don Diego de Mendoza, Secretario de Estado del rey de Portugal ${ }^{11}$, hizo levantar a Santa Cecilia ya que "es este señor devotísimo de esta santa y es el cuarto altar que la hizo, porque en todas las partes que ha estado la dejaba cultos, y en la nuestra [iglesia de las Trinitarias Descalzas] todos los años hacía fiesta"12. Estas palabras eran las que encabezaban un poema "Para cantar a santa Cecilia, en la fiesta que se hace en su altar, en el referido Convento de Trinitarias Descalzas", compuesto por una de las religiosas del Convento, Sor Francisca de Santa Teresa, cuyo manuscrito se conserva en éste ${ }^{13}$. Asimismo, el mecenazgo del altar para la capilla de Santa Cecilia por parte del Secretario de Estado del rey de Portugal Pedro II, se explica por el patrocinio que la casa de Braganza ejerció desde 1630 del convento e iglesia.

Para el ornato de dicha capilla se encargó un gran lienzo (fig. 1, 309 × 203 $\mathrm{cm}$ ) en el que se representaba a Santa Cecilia tocando el órgano custodiada por la presencia de multitud de ángeles. Los situados en la parte inferior tocan diversos instrumentos musicales, como la flauta, el violín, el contrabajo o la guitarra, y cantan con libretos en sus manos. Esos cánticos son acompañados por el coro angélico en la parte superior del lienzo que sustenta partituras

\footnotetext{
${ }^{8}$ Archivo del Convento de Trinitarias Descalzas de Madrid (ACTDM), Libro de Varios Papeles, fol. 194v., publicado por Pablo Cano Sanz, "Patrimonio pictórico en el convento de las Trinitarias Descalzas de Madrid: Estudio histórico-artístico de las cuatro capillas que ornamentan la nave del templo", Pátina, 16, (2011), p. 151 , doc. 10 .

9 ACTDM, Fray Francisco de Jesús, Libro sobre la Fundación e Historia del Convento, fols. 148-149, publicado por Cano Sanz, "Patrimonio pictórico...", p. 151, doc. 11.

10 ACTDM, Libro de Varios Papeles, fol. 293v, publicado por Cano Sanz, "Patrimonio pictórico...", p. 151, doc. 12.

${ }^{11}$ El cargo expreso de don Diego nos lo ofrece Pierre Massuet en su Historia del Duque de Riperdá, primer Ministro de España, en el Reynado del señor Felipe Quinto..., (Madrid: en la Imprenta de Josef López, 1796), p. 302.

12 Manuscrito de los poemas de Sor Francisca de Santa Teresa, fols. 303r-304r, publicado en María del Carmen Alarcón Román, Literatura conventual femenina en el Siglo de Oro. El Manuscrito de Sor Francisca de Santa Teresa (1654-1709). Estudio y Edición, (Sevilla: Tesis Doctoral, 2015), pp. 654-656.

13 Véase Alarcón Román, Literatura conventual..., pp. 654-656.
} 


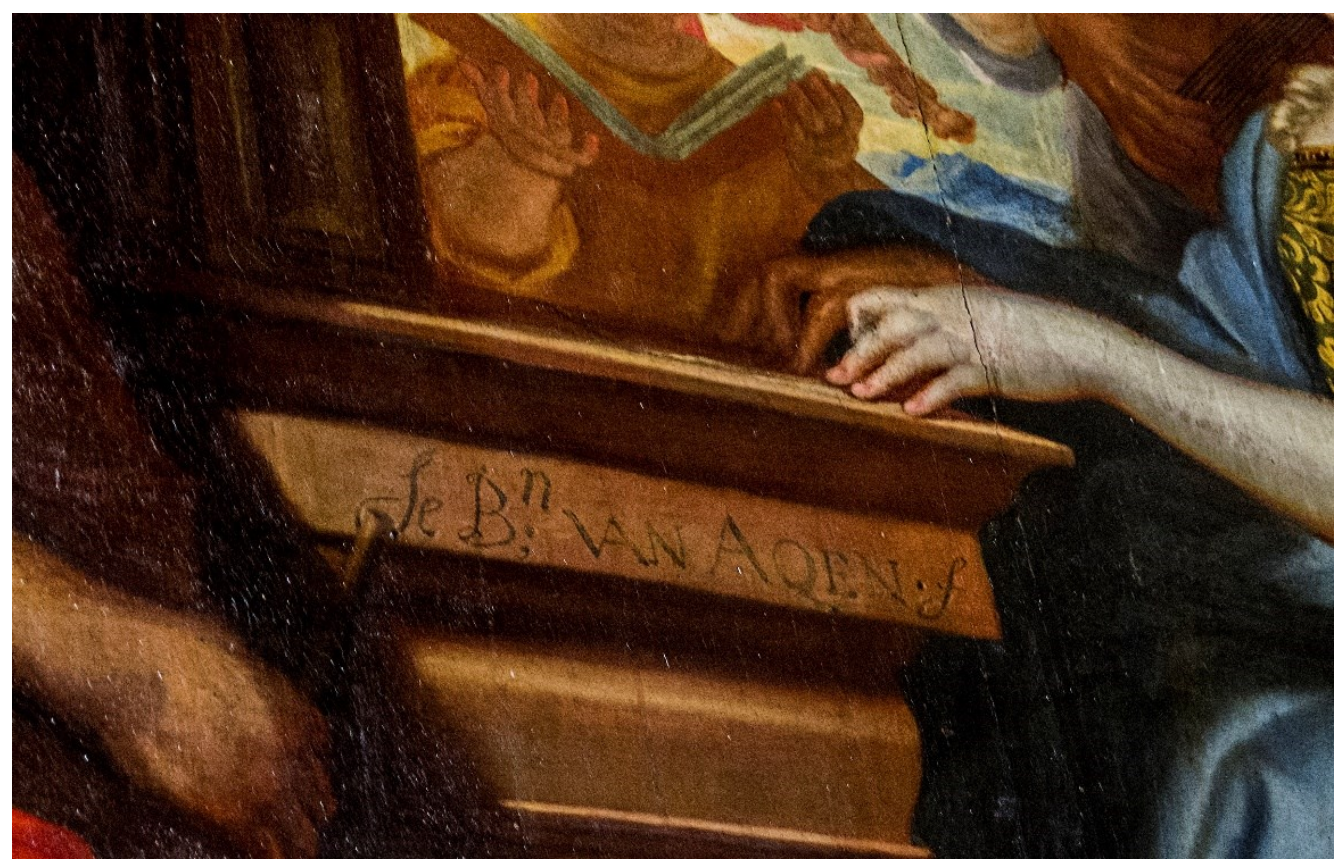

Fig. 2. Sebastiaen van Aken, Detalle de la firma en el cuadro de Santa Cecilia. Fotografía: Jesús Calleja Vicente $\subset$

entre sus manos. La obra está firmada en la parte baja del órgano por "Se $\mathrm{B}^{\mathrm{n}}$; VAN AQUEN, F" (fig. 2), inscripción que ya había sido recogida, pero leída incorrectamente como "Je $\mathrm{B}^{\mathrm{n} \text { " }}$ por otros historiadores ${ }^{14}$. Ésta hace referencia al artista flamenco Sebastiaen van Aken, bautizado en Malinas el 31 de marzo de 1648 y fallecido en su localidad natal el 21 de noviembre de $1722^{15}$.

Van Aken se dedicó principalmente a la realización de pintura religiosa. Fue discípulo de Lucas Franchoys el joven, también oriundo de Malinas, y quien formó parte del taller de Rubens hasta la muerte de éste en 1640. El 28 de diciembre de 1666, Van Aken obtuvo el nombramiento de maestro del gremio de San Lucas en Amberes ${ }^{16}$. Su primer trabajo tras ser nombrado maestro fue una gran Asunción para el priorato de Hanswyck en Malinas, sin embargo ésta parece que no gustó a los comitentes ${ }^{17}$. Ante el fracaso de esta obra decidió seguir ampliando su formación y marchar a Italia. Allí recaló en Roma donde está documentada su presencia entre 1676 y 1680 en el taller de Carlo Maratta ${ }^{18}$, donde aprendió los modos del maestro y su colorido. En

\footnotetext{
14 El apellido del pintor Van Aken, sin identificarse con ningún autor en concreto, había sido publicado correctamente por Ramón Guerra de la Vega, Iglesias y Conventos del Antiguo Madrid, (Madrid: 1996), p. 219. Poco después, Antonio de Vicente, consideraba que el lienzo estaba firmado "Van Aken o Van Owen", véase Antonio de Vicente Puente, Guía breve del Monasterio de S. Ildefonso y S. Juan de la Mata. MM. Trinitarias de Clausura, (Madrid: Gráficas Almeida, 1998), p. 13. Sin embargo, Cano Sanz, quién publica y transcribe la inscripción completa, fue quien leyó erróneamente el nombre del artista en la leyenda, no pudiendo dar con la autoría de la obra. Véase Cano Sanz, "Patrimonio pictórico...", p. 148.

15 Emmanuel Neefs, Histoire de la peinture et de la sculpture à Malines, (Gand: Imprimerie Eug. Vanderhaeghen, 1876), vol. I, pp. 392-393.

16 Neefs, Histoire de la peinture..., p. 392.

17 Jean Baptiste Deschamps, Voyage pittoresque de la Flandre et du Brabant: avec des réflexions relativement aux arts \& quelques gravures, (Paris: 1769), p. 132 y Neefs, Histoire de la peinture..., p. 393. 18 Neefs, Histoire de la peinture..., p. 393 y Carine Dechaux, "Van Aken, Sébastien", Le Dictionnaire des peintres belges du XIV siècle à nos jours, (Bruxelles: La Renaissance du livre, 1995), vol. II, p. 976.
} 


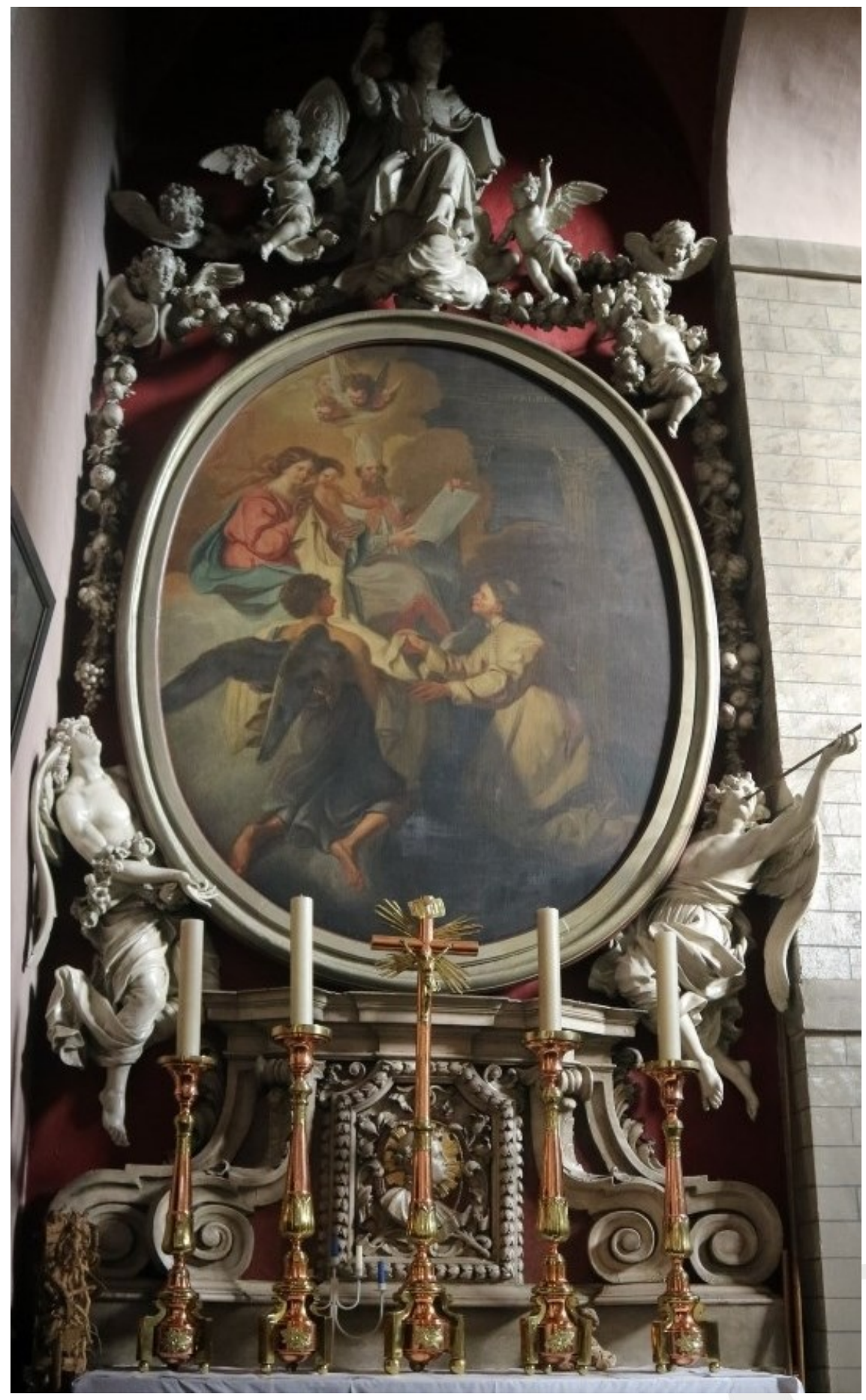

Fig. 3. Sebastiaen van Aken, Altar de San Norberto recibiendo de la Virgen el hábito de la Orden. Duffel, Parroquia de Onze-Lieve-Vrouw van Goede Wil. CIOED

Italia permanecerá al menos hasta el verano de $1697,{ }^{19}$ momento en el que parece iniciar viaje hacia España y Portugal. Se desconoce por completo la duración de este viaje y hasta ahora tampoco se sabía si había tenido actividad pictórica en ambos países. Lo que se conocía fehacientemente es

19 Godefridus Johannes Hoogewerff, Nederlandse Kunstenaars te Rome (1600-1725). Uittreksels uit de parochialen archieven, (La Haye: 1943), pp. 54, 153-155. 
que el 3 de junio de 1704 ya estaba de vuelta en su ciudad de origen, ya que se le pagaba en Malinas 26 florines por un retrato que Van Aken había realizado del rey de España ${ }^{20}$. Se desconoce si éste correspondía a Felipe $\mathrm{V} o$ era una efigie del archiduque Carlos, quien en 1702 había recibido el título de rey de España bajo el nombre de Carlos III $^{21}$. Desde ese momento el artista se dedicará a realizar encargos religiosos en los alrededores de Malinas hasta su fallecimiento en 1722 . De las obras que pintó tan sólo se había identificado hasta el momento un San Norberto recibiendo de la Virgen el hábito de la Orden que se conserva en Duffel, cerca de Malinas, en la Parroquia de OnzeLieve-Vrouw van Goede Wil (fig. 3), lienzo que como ahora veremos tiene similitudes tanto compositivas, presentando ambas una figura de espaldas en primer plano, como de colorido, gracias a la preeminencia de los tonos cálidos, con la Santa Cecilia de las Trinitarias Descalzas de Madrid.

La presencia del lienzo de Santa Cecilia con la firma de Sebastiaen van Aken en él acredita por tanto que el artista no sólo estuvo de paso por España, sino que también tuvo actividad pictórica durante el tiempo de su estancia hispánica. La obra de las Trinitarias debe fecharse entre julio de 1697, momento en el que se sabe que abandona Italia, y 1701 , ya que está documentado que el cuadro de la patrona de la música fue el último de los altares en ser instalado y con anterioridad a éste, el 6 de noviembre de $1701^{22}$, se había colocado el correspondiente a la Capilla de San Agustín el cual fue encargado a Juan de Espinosa de los Monteros, como consta en la inscripción que éste también posee. Es muy probable que, ante el competitivo ambiente artístico de la Italia de finales del siglo XVII, Van Aken estuviera intentando buscar fortuna en otro país europeo. España, y en concreto Madrid, gracias a la presencia de la Corte, era un lugar ideal para instalarse debido al gran número de coleccionistas y obras de tipo religioso que allí se promovían. De hecho desde la llegada de Luca Giordano en 1692 no habían dejado de llegar artistas extranjeros a la Villa, como es el caso de Jacques Courtilleau, hacia 1696, que se convertirá en pintor de Cámara de la reina Mariana de Neoburgo ${ }^{23}$; o el de John Closterman quien arribará de la mano del embajador británico Alexander Stanhope en 1698 con la intención de conseguir un puesto como retratista en la Corte y que, tras no conseguir su objetivo, marchará en la primavera de 1699 a Italia ${ }^{24}$.

Es seguro que la estancia de Van Aken en el taller de Carlo Maratta le abrió algunas puertas a su llegada a la Corte y fruto de ello debió ser el encargo del cuadro de la Santa Cecilia, oportunidad que el pintor aprovechó para in-

\footnotetext{
${ }^{20}$ Neefs, Histoire de la peinture..., p. 394 y Dechaux, "Van Aken...", vol. II, p. 976.

${ }^{21}$ Neefs cree que el cuadro podría corresponder con un retrato en óvalo, el cual parece perdido en la actualidad, que se conservaba en el Museo de Malinas a finales del siglo XIX. Véase Neefs, Histoire de la peinture..., p. 394.

22 Cano Sanz, "Patrimonio Pictórico...", p. 148.

23 Ángel Aterido, El final del Siglo de Oro. La pintura en Madrid en el cambio dinástico 1685-1726, (Madrid: CSIC, 2015), vol. II, pp. 82-83.

${ }^{24}$ Gloria Martínez Leiva, "Art as diplomacy: John Closterman's portraits of Carlos II of Spain and his wife Queen Maria Anna of Neuburg", Burlington Magazine, 1382, (2018), pp. 380-386.
} 


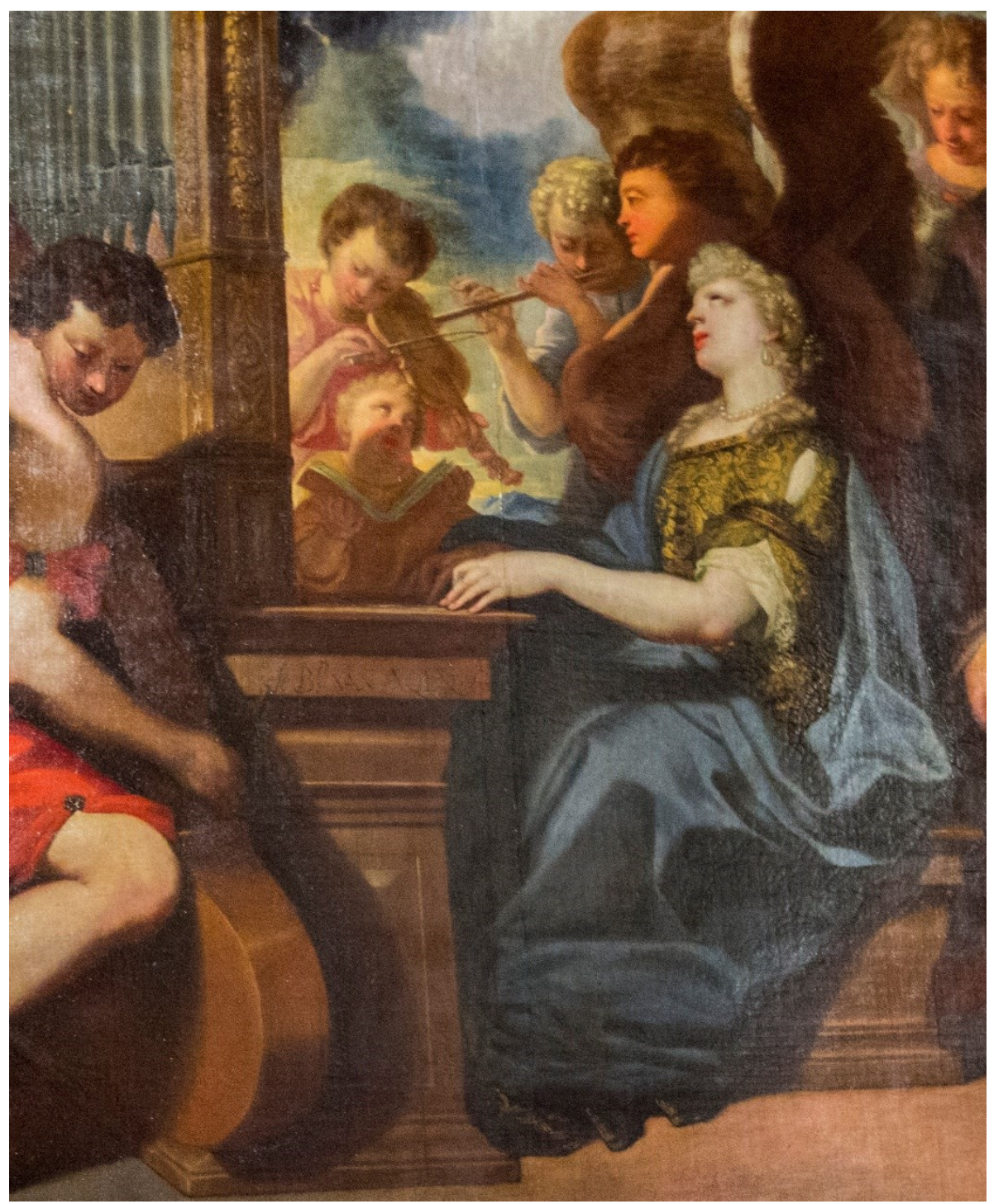

Fig. 4. Sebastiaen van Aken, Detalle de la figura de Santa Cecilia. Madrid, Iglesia del Convento de las Trinitarias Descalzas de Madrid, Fotografia: Jesús Calleja VicenteC

tentar dejar su carta de presentación, de ahí la gran firma de la obra, y hacerse un hueco artístico. En éste, de hecho, la personificación de Santa Cecilia como una mujer rubia, de piel blanquísima, ojos azules y con unos rasgos y atuendo semejantes a los que presentaba la reina Mariana de Neoburgo, podría indicar que el artista pretendía con esta obra no sólo hacer su carta de presentación en la Corte, sino también halagar a la soberana, conocida por su predilección por la música y su bonita $\mathrm{voz}^{25}$, encarnándola como a la Santa protectora de la música (fig. 4). Sin embargo, la difícil situación política que atravesaba España ante el deteriorado estado de salud

25 Sobre el gusto de la soberana por el arte musical hay diversos estudios específicos sobre el tema, como el de François Reynaud, "Mathieu Aubery, un Musicien Français a Bayonne au service de Marie-Anne de Baviere Neubourg: Les lamentations du Phophete Jeremie et le Psaume 50", Melanges de la Casa de Velázquez, 11 (1975), pp. 227-251 y Stephanie Klauk, "Durón y el entorno musical de Mariana de Neoburgo", en Sebastián Durón y la música de su época, eds. Paulino Capdepón y Juan José Pastor, (Pontevedra: Academia del Hispanismo, 2013), pp. 151-159. 
de Carlos II y por tanto su inminente muerte sin descendencia, circunstancias que hacían que el ambiente fuera sumamente tenso, no era el oportuno a la hora de conseguir un empleo en la Corte, tal y como le sucedió a John Closterman. Ante la imposibilidad de encontrar un hueco en Madrid, sería a través de su mecenas en las Trinitarias, don Diego de Mendoza y Corterreal, como el artista debió de recalar en Portugal en busca de mejor fortuna. Allí es donde conocerá a María Teresa Durant, joven de origen bruselense con la que contrajo matrimonio, siendo probablemente esto y la falta de encargos lo que le animó a volver definitivamente a su país ${ }^{26}$. De las obras que Van Aken pudo realizar durante su estancia en España y Portugal no tenemos más indicios, pero al menos la Santa Cecilia de las Trinitarias, que ahora ha podido agregarse a su catálogo, da buena muestra de la habilidad pictórica del artista y de su intento de establecerse como pintor en la Península Ibérica.

${ }^{26}$ Neefs, Histoire de la peinture..., p. 393. 
Bibliografía

Alarcón 2015: María del Carmen Alarcón Román, Literatura conventual femenina en el Siglo de Oro. El Manuscrito de Sor Francisca de Santa Teresa (1654-1709). Estudio y Edición, (Sevilla: Tesis Doctoral, 2015).

Aterido 2015: Ángel Aterido, El final del Siglo de Oro. La pintura en Madrid en el cambio dinástico 1685-1726, (Madrid: CSIC, 2015).

Cano 2011: Pablo Cano Sanz, "Patrimonio pictórico en el convento de las Trinitarias Descalzas de Madrid: Estudio histórico-artístico de las cuatro capillas que ornamentan la nave del templo", Pátina, 16 (2011), pp. 137-152.

De Vicente 1998: Antonio De Vicente Puente, Guía breve del Monasterio de S. Ildefonso y S. Juan de la Mata. MM. Trinitarias de Clausura, (Madrid: Gráficas Almeida, 1998).

Dechaux 1995: Carine Dechaux, "Van Aken, Sébastien", Le Dictionnaire des peintres belges du XIVe siècle à nos jours, (Bruxelles: La Renaissance du livre, 1995), vol. II, p. 976.

Deschamps 1769: Jean Baptiste Deschamps, Voyage pittoresque de la Flandre et du Brabant: avec des réflexions relativement aux arts \& quelques gravures, (Paris: 1769).

Guerra 1996: Ramón Guerra de la Vega, Iglesias y Convento del Antiguo Madrid, (Madrid: 1996).

Hoogewerff 1943: Johannes Hoogewerff Godefridus, Nederlandse Kunstenaars te Rome (1600-1725). Uittreksels uit de parochialen archieven, (La Haye: 1943).

Klauk 2013: Stephanie Klauk, "Durón y el entorno musical de Mariana de Neoburgo", en Sebastián Durón y la música de su época, eds. Paulino Capdepón y Juan José Pastor, (Pontevedra: Academia del Hispanismo, 2013), pp. 151-159.

Martínez 2018: Gloria Martínez Leiva, "Art as diplomacy: John Closterman's portraits of Carlos II of Spain and his wife Queen Maria Anna of Neuburg", Burlington Magazine, 1382, (2018), pp. 380-386.

Massuet 1796: Pierre Massuet, Historia del Duque de Riperdá, primer Ministro de España, en el Reynado del señor Felipe Quinto..., (Madrid: en la Imprenta de Josef López, 1796).

Menéndez 1921: Ramón Menéndez Pidal, "El convento de las Trinitarias de Madrid", Boletín de la Real Academia de la Historia, 79, (1921), pp. 97-99.

Neefs 1876: Emmanuel Neefs, Histoire de la peinture et de la sculpture à Malines, (Gand: Imprimerie Eug. Vanderhaeghen, 1876). 
Reynaud 1975: François Reynaud, "Mathieu Aubery, un Musicien Français a Bayonne au service de Marie-Anne de Baviere Neubourg: Les lamentations du Phophete Jeremie et le Psaume 50", Melanges de la Casa de Velázquez, 11 , (1975), pp. 227-251.

Tovar 1974: Virginia Tovar Martín, "El arquitecto Marco López y el Convento de las Trinitarias Descalzas de Madrid", Anales del Instituto de Estudios Madrileños, 10, (1974), pp. 133-153.

Tovar 1975: Virginia Tovar Martín, Arquitectos madrileños de la Segunda Mitad del siglo XVII, (Madrid: Instituto de Estudios Madrileños, 1975).

Tovar 1990: Virginia Tovar Martín, "El Monasterio de las Religiosas Trinitarias Descalzas de San Ildefonso de Madrid", Archivo Español de Arte, 251, (1990), pp. 401-418.

Recibido: 14/09/2018

Aceptado: 29/10/2018 